\title{
Reflexiones sobre \\ el financiamiento del desarrollo
}

\section{Roberto Frenkel}

Investigador Titular, CEDES

Director,

Banco de la Provincia

de Buenos Aires

frenkelr@bapro.com.ar

El artículo incluye reflexiones sobre la inserción financiera internacional de los países de la región y los diferentes segmentos de los sistemas financieros nacionales. En el marco del proceso de globalización financiera de América Latina, y sobre la base de las circunstancias que enfrentan los países más grandes de la región, se analizan diferentes factores que condicionan los escenarios financieros más allá de los argumentos estilizados de la sabiduría convencional: el riesgo de soberanía, el proceso de globalización financiera, el grado de integración financiera, el costo del capital y la gravitación de las primas de riesgo-país, la vinculación entre riesgo soberano y solvencia fiscal, y las consecuencias de la integración segmentada. Se consideran, asimismo, cursos de acción para reducir el riesgo-país. Por otro lado, se analiza el papel de los diferentes sectores institucionales en la generación del ahorro y se examinan las principales tendencias de la intermediación financiera en la región: la concentración bancaria, la mayor participación de entidades foráneas y el papel de la banca pública en el contexto actual. 


\section{Inserción financiera internacional}

\section{Introducción}

\section{a) El proceso de globalización financiera de Amé- rica Latina}

La inserción de América Latina en este proceso cumple veinticinco años, interrumpidos por la crisis de la deuda externa. A partir de comienzos del decenio de 1990 se produjo una reinserción y emergieron tendencias que sugerían que la región mostraba un proceso de integración creciente. La transitoriedad de los efectos tequila pareció confirmar esas tendencias. Sin embargo, después de la crisis asiática no se retornó a ese proceso. No se trata tan sólo de que las crisis no fueron "cortas", en el sentido de que sus consecuencias persisten en las economías que las sufrieron; también cabe interrogarse sobre las tendencias que manifestó el proceso de globalización financiera después de la crisis asiática. Además, han aparecido fenómenos novedosos, como la inestabilidad financiera en los Estados Unidos y su contagio a las economías emergentes de América Latina. Existe una inestabilidad financiera potencial de ese país que podría tener una repercusión importante sobre las economías más frágiles. Recientemente, la reducción de la tasa de interés estadounidense ha tenido un impacto favorable y se proyectan mayores reducciones con efectos semejantes. Este es un curso posible, pero hay que preguntarse también si es probable en el contexto post-1997, caracterizado por el "aprendizaje" que realizó el mercado en el último trienio y la ausencia de mayores innovaciones en las instituciones internacionales. También cabe abrir un interrogante sobre la magnitud de los renovados flujos de capital. ¿Puede esperarse una repetición del auge de ingresos de 1996-1997? Las necesidades de financiamiento de las mayores economías de la región exigen flujos de magnitud semejante a los recibidos en esa oportunidad, para alcanzar tasas de crecimiento significativas. Si no se repite ese auge, Brasil y Argentina encararán situaciones de gran fragilidad.

Este artículo se basa en material preparado para el Proyecto Conjunto CEPAL/BID sobre aspectos regionales del financiamiento del desarrollo en América Latina y el Caribe. b) Cambios en la inserción financiera de los países

Uno de los aspectos novedosos con relación al decenio de 1990 son los cambios experimentados en las economías receptoras. La inserción de los países ha ido cambiando. La ha ido modificando la acumulación de deuda externa e inversión extranjera directa (IED). Los balances de pagos tienen diferente estructura que al comienzo de la década. Los países altamente endeudados enfrentan como principal problema el refinanciamiento de sus deudas y el financiamiento de los déficit de cuenta corriente determinados por los servicios del capital (intereses y utilidades). El déficit de balance comercial ha perdido importancia relativa frente al déficit rígido y creciente en las cuentas de servicios financieros y factorales. En este aspecto, la situación se asemeja más a la de 1980 que a la de 1990.

Se puede analizar la diferente evolución de las economías en los años noventa en términos de trayectorias de integración financiera internacional. La noción subyacente es que se trata de un proceso con histéresis, en el cual las condiciones vigentes en un momento dependen de la historia previa. El ejemplo más evidente es la deuda externa. Ciertas trayectorias de integración financiera conducen a situaciones de mayor vulnerabilidad relativa, más propensas a las crisis. La CEPAL ha hecho hincapié en este aspecto y ha analizado políticas para evitar esas trayectorias. Sus recomendaciones están en buena medida fundadas en el análisis comparado de las trayectorias que siguieron los países, las distintas políticas que confluyeron para determinarlas y la experiencia que proporcionan los episodios de crisis (por dinámica propia o por mayor propensión al contagio). Pero los países que siguieron las trayectorias conducentes a mayor vulnerabilidad, aunque hayan tenido y superado crisis financieras y cambiarias, exhiben hoy, como consecuencia de su trayectoria pasada, situaciones "estructurales" de mayor vulnerabilidad (relaciones deuda externa/PIB, déficit en cuenta corriente/PIB, deuda externa/exportaciones, déficit en cuenta corriente/exportaciones, estructura de la cuenta corriente, estructura del sistema financiero).

Las altas primas de riesgo-país que enfrentan esas economías resultan de la apreciación que realiza el mercado de estas condiciones de mayor vulnerabilidad relativa. Al mismo tiempo, dicha apreciación tiende a 
preservar o acentuar esas condiciones, por los efectos de las altas tasas de interés y menores flujos de capital sobre el crecimiento, el sector externo y las condiciones del sector financiero. Estos países están estacionados en una trampa de financiamiento. Son más propensos a las crisis por desencadenantes internos o por contagio, pero su situación se ha prolongado sin transformarse en una crisis cambiaria y financiera.

Brasil, por ejemplo, corrigió después de la crisis varios de los elementos que configuraron su trayectoria previa (devaluación, establecimiento de un régimen cambiario de flotación sucia, ajuste fiscal). Sin embargo, el país no podía cambiar la herencia "estructural" de su trayectoria previa (por ejemplo, sus relaciones de endeudamiento y el componente predeterminado de su cuenta corriente). El mercado evalúa estas condiciones y aplica una prima de riesgo-país alta, y la economía, pese a que en el año 2000 tuvo un crecimiento, sigue aprisionada en su trampa de financiamiento.

Argentina superó su crisis de finales de 2000 con un rescate internacional, pero sin ningún cambio de política con relación a la que determinó su trayectoria anterior (ésta incluía en el año previo un ajuste fiscal contractivo que no tuvo efecto sobre la prima de riesgo). El mercado obtuvo un reaseguro en el corto plazo, pero la prima de riesgo siguió reflejando condiciones de entrampamiento.

En lo que sigue se ponen de relieve factores que condicionan los escenarios enfrentados por los mayores países de la región, ${ }^{1}$ más allá de los argumentos estilizados de la sabiduría convencional.

\section{Más allá de los argumentos estilizados}

\section{a) El riesgo de soberanía}

La frontera nacional define una jurisdicción política y legal. Dentro de esta jurisdicción rige la soberanía del gobierno y otras instituciones del Estado nacional. En determinadas circunstancias, las autoridades de una nación pueden decidir o avalar el incumplimiento de ciertos contratos, aspecto de la soberanía que limita la capacidad de un agente económico extranjero de hacer cumplir el contrato que lo involucra. Este es un riesgo de soberanía irreductible. No hay razones para suponer a priori que el valor de este riesgo resulte muy significativo, pero hay una tensión entre el

\footnotetext{
${ }^{1}$ Reflejan, principalmente, las circunstancias que enfrentan Argentina, Brasil y, en menor medida, México, pero en algunos casos son también aplicables a otros países de la región.
}

proceso de globalización financiera y la institucionalidad de los Estados nacionales que puede resultar en situaciones de integración financiera segmentada.

\section{b) El proceso de globalización financiera}

Este proceso está cerca de cumplir tres décadas. Parece razonable fechar su comienzo entre 1971 y 1973, cuando Estados Unidos liberó la atadura del dólar al oro y se adoptó la flotación de las monedas de los principales países desarrollados. Desde entonces tuvo lugar una secuencia de liberalizaciones y desregulaciones de los movimientos internacionales de capital y de los sistemas financieros nacionales. La competencia en el mercado desempeñó un importante papel impulsor, de modo que los procesos de liberalización de los flujos financieros entre países y de los sistemas nacionales se incentivaron mutuamente. La emergencia de nuevos negocios internacionales puso presión para lograr la reducción de costos y menores regulaciones en el plano nacional. A la inversa, nuevas oportunidades abiertas en algunos países impulsaron la desregulación de las transacciones entre países. En paralelo con la secuencia de reformas hubo un rápido crecimiento del volumen de transacciones financieras a través de las fronteras.

Este proceso de creciente integración involucró e involucra principalmente a los países desarrollados. Sin embargo, las mayores economías de América Latina fueron parte de él desde sus primeros tiempos. Brasil primero y más adelante México, Venezuela, Argentina y Chile fueron importantes receptores de capital en el decenio de 1970. Los dos últimos, junto con Uruguay, fueron entonces pioneros de drásticas reformas liberalizantes que anticiparon las que se generalizaron en el decenio de 1990.

La participación de América Latina en la globalización financiera sufrió una interrupción con la crisis de la deuda de los años ochenta. La crisis impuso un hiato de unos ocho años, en los cuales desapareció el financiamiento voluntario. En los años noventa — digamos desde la firma del Plan Brady por parte de México- América Latina se reincorporó vigorosamente al proceso de globalización en sus dos planos, a través de drásticas reformas liberalizantes y crecientes flujos (y reflujos) de capital.

\section{c) El grado de integración financiera}

En las formulaciones de las tempranas experiencias del Cono Sur y de manera más generalizada en los años noventa, la integración financiera internacional fue el horizonte manifiesto de quienes promovieron 
intelectualmente el proceso. La integración completa equivale al establecimiento de una intermediación financiera global en la cual el rendimiento de las colocaciones del público, por un lado, y el costo del capital de quienes toman los créditos, por otro, se igualan para las transacciones económicamente equivalentes (plazos, riesgos, garantías, etc.), con independencia de la localización geográfica de ahorristas y deudores.

La integración completa supondría minimizar los costos de intermediación, reducir el costo del capital al nivel de los países desarrollados y, en la medida en que nuestro menor desarrollo relativo implica mayores oportunidades de nuevos negocios, aseguraría las corrientes de inversión y financiamiento que tenderían a disminuir la brecha de desarrollo.

En comparación con el aislamiento financiero que rigió desde la crisis del treinta hasta avanzados los años sesenta, es indudable que el proceso de globalización alcanzó un significativo grado de integración financiera entre los países desarrollados y también entre éstos y los que se constituyeron como mercados emergentes. Sin embargo, la avanzada integración financiera entre países desarrollados aún está lejos de ser completa. La igualación de las tasas de interés nominales sólo se verifica en los casos de operaciones aseguradas en los mercados de cambios futuros. Las tasas de interés reales no tienden en general a igualarse. Los ciudadanos de cada país exhiben una marcada preferencia por activos de su patria. Las tasas de inversión muestran una alta correlación con las tasas de ahorro nacionales. En síntesis, aunque han alcanzado un alto grado histórico de integración, los mercados financieros de los países desarrollados continúan exhibiendo una significativa diferenciación.

Menor aún es el grado de integración financiera entre países desarrollados y subdesarrollados. No sólo porque la globalización involucra a una proporción pequeña de países con "mercados emergentes", sino también por las características propias de la integración de estos mercados. Señalemos, en primer lugar, que aun en los momentos de auge, el volumen de los flujos financieros es muchísimo menor de lo que teóricamente cabría esperar de una integración completa. En los países desarrollados, la inversión en mercados emergentes está concentrada en agentes especializados y representa una pequeña proporción de los activos de sus residentes.

\section{d) Una integración segmentada}

Para las mayores economías de la región, la mencionada reincorporación de América Latina al proceso de globalización en los años noventa tuvo como primer acto la conversión en bonos Brady de la deuda externa pública de arrastre, efectuada en las negociaciones de los años ochenta. El reingreso al mercado voluntario fue simultáneo con la flotación de una importante masa de bonos públicos cuya tenencia se diversificó en un activo mercado secundario. Los bonos de deuda pública se constituyeron así en el basamento del nuevo mercado de inversiones en la región desde su inicio. Ese mercado de deuda pública se engrosó posteriormente con las emisiones que realizaron los gobiernos.

Como representan compromisos en dólares, el único riesgo que conllevan los bonos de deuda externa pública es el de incumplimiento. El valor que el mercado atribuye a este riesgo (la prima de riesgo-país o prima de riesgo soberano) se mide como la diferencia entre el rendimiento que se obtendría comprando el bono a su precio actual y el rendimiento resultante de adquirir un bono de semejantes características financieras del gobierno de los Estados Unidos, el deudor en dólares que ofrece menor riesgo de incumplimiento.

La evolución de las primas de riesgo soberano no muestra evidencias de que el sistema internacional que se ha ido conformando con la globalización tienda a una integración financiera completa. Por el contrario. La experiencia de los recientes tres años - el período que se inicia con la crisis asiática - sugiere que el sistema ha conformado una integración segmentada, en la cual el costo del capital para las economías emergentes de América Latina es sistemáticamente mucho mayor que en los países desarrollados.

\section{e) Las primas de riesgo país}

Una tendencia convergente a una integración financiera completa hubiera supuesto una continua reducción de las primas de riesgo-país de América Latina. No ha ocurrido así. Tomamos como ejemplo la evolución en los años noventa de la prima (medida por el EMBI+) de Argentina, la economía financieramente más abierta y desregulada de la región. La prima nunca cayó por debajo de un promedio mensual de 280 puntos básicos y sólo tocó ese mínimo en un par de ocasiones. Luego de reducirse desde principios de los noventa, alcanzó ese mínimo en los primeros meses de 1994, para retomar una tendencia ascendente a partir de marzo de ese mismo año, cuando Estados Unidos elevó sus tasas de interés. Luego, el promedio mensual se disparó a 1800 puntos básicos con el efecto tequila. En seguida descendió gradualmente para tocar otra vez el mencionado mínimo en el mes previo a la devaluación de Tailandia. Desde la crisis asiática en adelante, los promedios mensuales nunca cayeron por debajo de los 400 puntos básicos, y superaron los 1000 con las 
crisis rusa y brasileña. En 1999 y 2000, sin nuevas crisis financieras y cambiarias nacionales, la prima nunca descendió por debajo de los 500 puntos básicos, y en el año 2000 tendió a incrementarse en correlación con otros impulsos, primero con la caída del NASDAQ y luego con el aumento del precio del petróleo.

El gráfico 1 muestra los promedios mensuales de las primas de riesgo de Argentina, Brasil, Chile y México, medidas por el Emerging Markets Bonds Index Plus, del banco J.P. Morgan (EMBI+). El nivel relativo de las primas de riesgo de las economías emergentes latinoamericanas está asociado con ciertas características estructurales de ellas, indicativas de su solvencia. Por ejemplo, como indica el cuadro 1 , se observa que los niveles recientes de las primas de México y Chile, por un lado, y Argentina y Brasil, por otro, están asociados con las respectivas relaciones deuda externa/exportaciones. También cabe mencionar que ingredientes locales, como los episodios de incertidumbre política en Argentina, influyen en la variación relativa de las primas latinoamericanas. Pero al trazar su evolución a lo largo de los años noventa aparece nítida la fluctuación común asociada a los episodios de crisis nacionales y, más recientemente, a brotes de incertidumbre de otro origen en el mercado de los países desarrollados.

\section{f) Contagio y movimientos de manada}

Tales fluctuaciones comunes son resultado de movimientos de manada de los inversores. La posibilidad misma de estos movimientos era desacreditada en la primera mitad de los años noventa por la ortodoxia
CUADRO 1

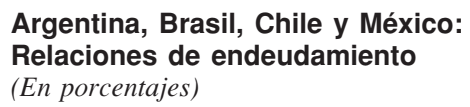

(En porcentajes)

\begin{tabular}{|c|c|c|c|}
\hline Años & $\begin{array}{c}\text { Exportaciones }^{\mathrm{a}} \\
\text { / PIB }\end{array}$ & $\begin{array}{c}\text { Deuda externa }^{\mathrm{b}} \\
\text { / PIB }\end{array}$ & $\begin{array}{l}\text { Deuda externa }{ }^{b} \\
\text { / exportaciones }\end{array}$ \\
\hline \multicolumn{4}{|c|}{ Chile } \\
\hline 1997 & 28.1 & 35.2 & 1.3 \\
\hline 1998 & 26.7 & 43.4 & 1.7 \\
\hline 1999 & 29.0 & 50.8 & 1.8 \\
\hline \multicolumn{4}{|c|}{ Brasil } \\
\hline 1997 & 7.6 & 25.5 & 3.4 \\
\hline 1998 & 7.4 & 30.1 & 4.0 \\
\hline 1999 & 10.2 & 44.1 & 4.3 \\
\hline \multicolumn{4}{|c|}{ México } \\
\hline 1997 & 30.4 & 38.1 & 1.3 \\
\hline 1998 & 30.8 & 38.3 & 1.2 \\
\hline 1999 & 30.7 & 33.4 & 1.1 \\
\hline \multicolumn{4}{|c|}{ Argentina } \\
\hline 1997 & 10.6 & 42.6 & 4.0 \\
\hline 1998 & 10.4 & 47.2 & 4.5 \\
\hline 1999 & 9.8 & 51.1 & 5.2 \\
\hline 2000 & 11.0 & 52.0 & 4.7 \\
\hline
\end{tabular}

a Exportaciones de bienes y servicios reales.

b Deuda externa de los sectores público y privado.

que predominaba entonces tanto en los organismos internacionales y los gobiernos como entre los analistas. Pero ese diagnóstico obtuvo reconocimiento con la crisis de México y se impuso como una realidad indiscutible con la crisis asiática y sus continuaciones.

La noción ganó status en el concepto de contagio, idea que ahora está incorporada en el diagnóstico

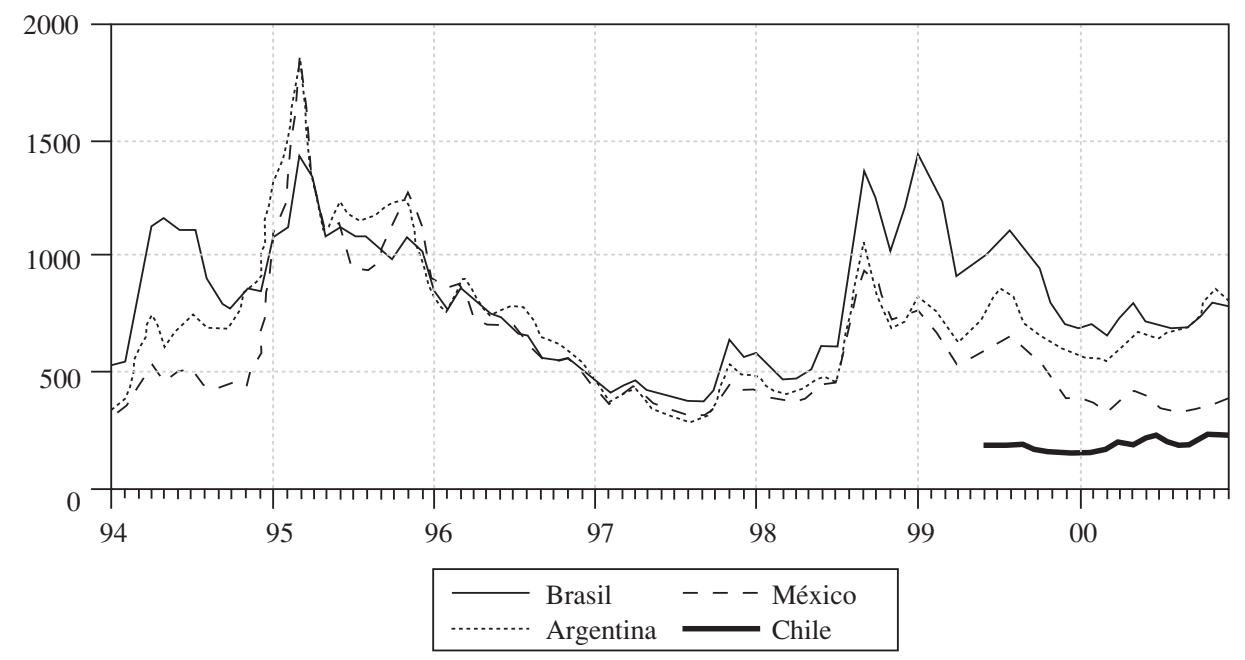


del Fondo Monetario Internacional (FMI) y fundamenta algunas de sus nuevas líneas de acción. Sin embargo, este concepto se limita a caracterizar los movimientos de manada inducidos por crisis nacionales. ¿Pero no representa un contagio semejante el detonado por la caída de las acciones tecnológicas que agrupa el NASDAQ, al cabo de una prolongada burbuja? ¿No cabe en el mismo concepto el efecto observado del aumento del precio del petróleo? El impacto de este aumento sobre la prima de México ilustra este último punto: aunque el alza del precio del petróleo benefició a la economía mexicana, su prima de riesgo soberano subió junto con las del resto de la región.

El reconocimiento de que también los fenómenos mencionados son de contagio es importante para el diseño y la promoción de medidas internacionales a favor de la estabilidad y el mejor funcionamiento del mercado globalizado, como argumentaremos más adelante.

Podemos imaginar cualquier escenario para el futuro - desde una crisis financiera generalizada y el retorno al aislamiento y la negociación de las deudas externas, hasta una estabilización de los mercados y la gradual convergencia a una integración financiera completa- porque es verdad que el futuro es irremediablemente incierto. Pero antes de imaginar el futuro debe reconocerse como un hecho que al cabo de una década de la reinserción financiera de América Latina, en los tres años transcurridos desde la crisis asiática las primas de riesgo-país han determinado que el costo de capital de las colocaciones gubernamentales de Brasil y Argentina, en las mejores condiciones de corto plazo experimentadas en el período, doblen aproximadamente la tasa de interés de los Estados Unidos y sea significativamente más alta que la de México. En América Latina, sólo la prima de Chile es semejante a las de la República de Corea, Malasia, Polonia y Hungría, los mercados emergentes con menor prima de riesgo soberano.

\section{g) El riesgo soberano se extiende más allá de la solvencia fiscal}

Podría pensarse que bastaría tener equilibrio fiscal y no requerir nuevas colocaciones para esterilizar los efectos del riesgo soberano. Podría argumentarse, y muchos lo hacen, que todo el problema reside en las finanzas del sector público. No es así. Chile, por ejemplo, tiene superávit fiscal, pero su prima no es despreciable. ¿En qué reside el riesgo soberano en un caso así? Las finanzas de un país pueden estar equilibradas o arrojar superávit, pero eso no garantiza que su eco- nomía disponga de los recursos en moneda extranjera necesarios para atender los servicios y amortizaciones de la deuda en dólares. Más aun, es posible que el gobierno cuente con los recursos necesarios en moneda extranjera para satisfacer sus propios requerimientos, pero no así los del conjunto de la economía, de modo que podrían faltar los dólares necesarios para servir la deuda externa privada. En estas condiciones las autoridades pueden, por elección o por verse obligadas a ello, suspender la convertibilidad de la moneda nacional (o los pagos al exterior, en el caso de una economía dolarizada) y forzar el incumplimiento de los contratos. La soberanía faculta esta posibilidad. El riesgo soberano se extiende más allá del riesgo de insolvencia fiscal.

\section{h) La prima de riesgo-país determina el costo del capital para la economía \\ La tasa de interés en dólares que ofrecen los títu-} los de deuda pública transados en el mercado secundario proporciona a todo el mercado una medida del riesgo soberano y tiende a determinar el costo del capital de las actividades del país, en moneda extranjera y en moneda local. En primer lugar, es el costo de oportunidad del capital de la inversión extranjera directa. En segundo lugar, establece un piso para el costo de captación de recursos internacionales por parte de las empresas nacionales. En tercer lugar, fija el piso del costo de captación internacional de los bancos y, por lo tanto, el piso del costo marginal de quienes se financian localmente en moneda extranjera. Por último, también tiende a determinar el piso del costo del capital en moneda local. Una breve elaboración sobre este último punto dará pie para señalar otras particularidades de la integración segmentada.

Indicamos más arriba que los inversores locales de los países desarrollados prefieren activos de su propio país, denominados en su propia moneda. Aun con un alto grado de interconexión financiera internacional, esta preferencia permite, en principio, que sus autoridades monetarias instrumenten políticas tendientes a establecer una tasa de interés en moneda local sistemáticamente inferior a la tasa internacional (esto es, a la tasa que el inversor obtendría de una colocación en moneda extranjera). En nuestras economías, por varias razones que no cabe puntualizar aquí, las preferencias son las inversas. Como la apertura financiera funciona en ambas direcciones, los agentes locales están habilitados para arbitrar entre activos en moneda local y dólares. Por esta razón, salvo en el caso (excepcional) de una tendencia sistemática y predecible 
a la apreciación de la moneda local, la tasa de interés real en moneda local debe ser a lo menos igual y generalmente mayor que la tasa de interés en dólares.

\section{i) Consecuencias de la integración segmentada}

La persistencia de altas primas de riesgo-país es un efecto impensado de la globalización financiera. Tiene varias consecuencias negativas. En primer lugar, las tasas de interés altas reducen la inversión y representan un freno al crecimiento. En segundo lugar, determinan una tendencia regresiva en la distribución del ingreso. En tercer lugar, imponen una transferencia de renta al exterior, directamente a través del servicio de la deuda externa e indirectamente a través de las utilidades de la inversión extranjera directa. Por último, en algunos casos —notablemente Argentina y Brasilimplican trayectorias macroeconómicas insostenibles, por la tendencia explosiva de las obligaciones de la deuda externa.

Frente a esta situación podría sugerirse volver atrás, desembarcar al país del proceso de globalización financiera. Pero no parece existir un camino sencillo para instrumentar esta idea. La deuda externa pública y privada es actualmente la principal ancla con el sistema financiero internacional. El servicio regular de la deuda absorbe gran parte de los ingresos brutos de capital.

A principios de los años noventa los países tenían cierto margen de elección del tipo y grado de apertura financiera, frente a la presión de fuertes ingresos de capital. La situación es hoy completamente distinta para varios países, cuyo problema es ahora principalmente el de obtener financiamiento para el giro regular de la deuda, con la imperiosa necesidad de obtenerlo a precios menores que los que se enfrentan. Consideremos los ejemplos de Brasil y Argentina. A principios de los años noventa Brasil contaba con un superávit comercial de unos 12000 millones de dólares y un balance de cuenta corriente equilibrado. Ensayaba entonces medidas para frenar los ingresos de capital, por sus efectos monetarios desestabilizantes. En 1999, luego de haber pasado por un proceso de estabilización y apertura comercial y financiera y tras haber transcurrido un año desde la crisis y las medidas correctivas, las cuentas externas de Brasil muestran un balance comercial aproximadamente equilibrado y un déficit de cuenta corriente del orden de los 25000 millones de dólares, por intereses y servicios de factores. Entre principios de los años noventa y 1999, el saldo de la cuenta de servicios por intereses, utilidades y dividendos pasó de $22 \%$ a $40 \%$ de las exporta- ciones de bienes. Análogamente, Argentina a principios de los noventa exhibía un déficit comercial de unos 2000 millones de dólares y un déficit de cuenta corriente de 6000 millones. En 1999, en el piso de la recesión, el balance comercial arrojó un déficit de 700 millones de dólares, mientras el déficit de cuenta corriente alcanzó a 12300 millones, por intereses y servicios del capital. El saldo de la cuenta de intereses y servicios del capital, como proporción de las exportaciones de bienes, pasó de $20 \%$ a $33 \%$ entre principios de los años noventa y 1999.

\section{j) Equilibrios múltiples}

Los niveles relativos de las primas de riesgo reflejan la apreciación que hace el mercado de distintos grados de vulnerabilidad y están correlacionados con indicadores de solvencia, como señalamos más atrás. La mayor vulnerabilidad relativa implica una mayor propensión a la crisis, frente a una perturbación (shock) equivalente. La ocurrencia de una crisis, cualquiera sea el factor desencadenante, puede racionalizarse como el paso de un equilibrio a otro en un modelo de equilibrios múltiples (ésta es la principal aplicación de los modelos de equilibrios múltiples en la literatura sobre el tema). Cualquier economía puede sufrir una crisis frente a una perturbación de suficiente entidad, pero hay configuraciones más propensas a experimentar crisis (a "saltar" de su equilibrio presente a una situación de crisis). De esta manera, todas las economías tienen dos "equilibrios": el equilibrio presente ( $\sin$ crisis, el bueno) y el equilibrio de la crisis. En algunas economías el equilibrio presente es más inestable, esto es, exhibe mayor vulnerabilidad.

Lo expuesto puede enriquecerse con dos consideraciones. La primera es que se puede razonar en términos de trayectorias, calificadas de acuerdo a sus potenciales de crecimiento y sostenibilidad, en lugar de concebir como un único "equilibrio" a cualquier situación no crítica. La economía puede situarse por un período más o menos prolongado en una "trampa" de altas tasas de interés, bajo crecimiento y alta vulnerabilidad. Está así sobre una trayectoria no sostenible a largo plazo (por la tendencia explosiva de las relaciones de endeudamiento), pero puede desenvolverse en esta situación por un cierto período sin enfrentar efectivamente una crisis. Esta configuración es el resultado conjunto de cierta trayectoria previa de integración financiera internacional y de la apreciación que hace el mercado de sus riesgos. Así, en lugar de distinguir entre dos equilibrios (crisis y no crisis) podemos distinguir entre dos trayectorias: la configuración 
de una trampa de bajo crecimiento y una trayectoria de crecimiento virtuoso.

La segunda consideración es que la ocurrencia o no de crisis y el tipo de trayectoria sobre la que evoluciona la economía no son independientes de la apreciación que hace el mercado, esto es, de la prima de riesgo que demanda y del volumen de activos del país que está dispuesto a absorber. Por ejemplo, a igualdad del resto de las condiciones, una economía puede encontrarse sobre una trayectoria de trampa o sobre una de crecimiento virtuoso, según la prima de riesgo-país y el flujo de capitales que recibe. La economía puede haber caído en una trayectoria de trampa por un efecto de contagio, pero una vez situada en esta posición sus indicadores tienden a empeorar y la apreciación negativa del mercado es una profecía autocumplida. La baja de la prima de riesgo-país y el mayor flujo de financiamiento podría reponer un crecimiento virtuoso, pero los inversores no modificarán sus expectativas sin una señal coordinadora.

Con la perspectiva de equilibrios múltiples, una acción internacional para reducir el riesgo soberano (por ejemplo, la presencia de una función de prestamista de última instancia) puede racionalizarse como una acción preventiva de la crisis, pues reduce la propensión a saltar al equilibrio crítico. Igualmente, la distinción entre tipos de trayectoria permite racionalizar la intervención como la acción necesaria en determinados casos para (generar la posibilidad de) que la economía salga de una situación de trampa de bajo crecimiento y pase a una trayectoria de crecimiento virtuoso.

\section{Cursos de acción para reducir el riesgo-país}

El fundamento más profundo de las primas de riesgo soberano es precisamente la soberanía de la cual gozan las naciones en nuestro tiempo. El proceso de globalización financiera podría haber dado otros resultados, pero la situación en que se encuentra estaba dentro de los cursos probables. Frente a los hechos que configuran la integración segmentada solemos razonar por analogía con los sistemas financieros nacionales para señalar las fallas del sistema conformado por la globalización. Observamos que se configuró un sistema internacional en el cual están ausentes gran parte de las instituciones que fueron construyéndose a lo largo del tiempo en los sistemas nacionales, para mejorar su funcionamiento y darles estabilidad. Estas instituciones y experiencias nacionales sugieren la conveniencia de diseñar instituciones que desempeñen funciones análogas en el plano internacional, para cuyo establecimiento se necesita en cada caso la cesión de distintos atributos de la soberanía nacional. Ocurre así incluso en lo que respecta a la producción y disponibilidad de información fiscal y financiera, que es el área en que más se ha avanzado en las discusiones y acuerdos de los foros de la Arquitectura Financiera Internacional (AFI). Y mucho más si se trata de establecer en el plano internacional instituciones de supervisión y regulación prudencial, tema en el que ha habido poco avance y ante el cual expresaron renuencia algunos países en desarrollo, temerosos de que las previsiones por riesgo pudieran restringir los ansiados flujos de capital. Los debates internacionales de la AFI son importantes, pero creemos que no cabe esperar que de ellos surjan soluciones en un plazo corto. Habría que buscar otros caminos.

En tanto el riesgo soberano es esencialmente uno de incumplimiento de contrato motivado por la imposibilidad práctica de afrontarlo, tiende a reducirlo la existencia de garantías adicionales en la forma de fondos de acceso contingente, a disposición de países que enfrentan dificultades. Esta función es análoga a la de prestamista de última instancia que cumplen en el plano nacional los bancos centrales de muchos países. Cabe imaginar muchas instituciones que puedan desempeñar esta función y las Naciones Unidas debería continuar impulsando el debate sobre el tema en los foros de la AFI. Pero considerando las posiciones que venía sosteniendo Estados Unidos y las que sostendrá probablemente su nuevo gobierno, parece claro que las funciones financieras internacionales seguirán concentrándose en el plazo previsible en las instituciones de Bretton Woods.

El instrumento del FMI que más se acerca formalmente a la función de provisión de garantías para reducir el riesgo soberano es la línea de crédito contingente, recientemente reglamentada. Sin embargo, las condiciones de acceso a esa línea son tan exigentes que los países que pueden cumplir con tales condiciones no se sienten inducidos a recurrir a ella, mientras que quienes más la necesitan no reúnen los requisitos. En paralelo, el Fondo ha establecido otra línea de crédito -el servicio de reservas suplementarias- de monto no definido y con condiciones de acceso mucho más discrecionales. La creó en la operación de rescate de la República de Corea, la aplicó en las operaciones relacionadas con las crisis de Rusia y Brasil y recientemente la concedió a la Argentina.

Con la creación de esta última línea, el FMI ha dado un paso significativo en la dirección de acercar 
su función a la de un prestamista de última instancia en el plano internacional. Parecería razonable procurar el desarrollo de esta línea como el camino más viable, aunque no debería abandonarse la discusión de las condiciones de acceso a la línea de crédito contingente. Para poder dar pasos adicionales hacia una reducción significativa de las primas de riesgo habría que aumentar los fondos disponibles y ampliar las circunstancias en que se puede acceder a ellos. Se lograría un acceso más abierto, por ejemplo, si se ampliara la noción de contagio, reconociendo los efectos derivados de brotes de incertidumbre de distintas fuentes en el mercado financiero desarrollado, como señalamos más atrás.

Una mayor disponibilidad de fondos — de origen público u obtenidos en los mercados con garantía indirecta de los gobiernos de los países desarrolladosy condiciones de acceso más amplias y automáticas podrían tener un efecto significativo sobre las primas de riesgo y mejorarían el funcionamiento del sistema porque reducirían las probabilidades de crisis. Pero esas condiciones difícilmente podrán lograrse sin cesiones adicionales de soberanía a los organismos multilaterales, precisamente porque esa cesión de soberanía opera conjuntamente con la mayor disponibilidad de fondos contingentes para reducir el riesgo soberano. Un ejemplo de este tipo de efecto es la nula prima de riesgo que paga la economía de Grecia, por ser un país miembro de la Unión Europea.

En teoría, ceder soberanía a los organismos multilaterales no significa perderla, sino ejercitarla en forma compartida, negociada. Sin embargo, el FMI y el Banco Mundial no están organizados democráticamente. Las cesiones de soberanía sin obtener como contrapartida un incremento de la participación de los países cedentes en el gobierno de los organismos están destinadas a confrontar una creciente (y legítima) conflictividad. En consecuencia, junto con la orientación tendiente a ampliar las funciones de prestamista de última instancia indicadas arriba, la agenda debería incluir en forma prioritaria el tema del gobierno de los organismos multilaterales.

\section{II}

\section{Sistemas financieros nacionales}

A lo largo del decenio de 1990 los países de América Latina han impulsado, en mayor o menor grado, la agenda de política económica inspirada en el llamado Consenso de Washington. El sustento teórico de las medidas fue aportado por una vasta literatura, cuyas conclusiones respaldaban fuertemente un enfoque amigable con el mercado para la solución de los principales problemas económicos de la región, entre ellos los vinculados con el sistema financiero. Pero los resultados de su aplicación han estado con frecuencia muy lejos de las expectativas generadas por sus propulsores, e incluso a veces fueron de signo contrario al esperado. Además, no se tuvo en cuenta cuán específicos son los problemas de cada país, pasando por alto en ocasiones "detalles" de estructura institucional de suma importancia.

Por otro lado, las políticas aplicadas no incorporan una lectura objetiva de experiencias internacionales relevantes fuera de la región. Existen ciertas lecciones que puedan ser extraídas, por ejemplo, de los procesos de largo plazo de los países del sudeste asiático. Si bien ciertas estrategias allí aplicadas, como el uso masivo de mecanismos de ahorro forzoso y/o políticas de fomento sectorial bajo un sistema eficaz de premios y castigos, aparecen como difícilmente replicables en los actuales contextos económicos y políticos de América Latina, entendemos que no deben ser descartadas de plano, en especial si se tiene una visión escéptica acerca de los resultados de las políticas aplicadas en el decenio de 1990.

\section{El ahorro}

La literatura sobre el tema admite las dificultades que existen para determinar con cierto grado de precisión cuál es el impacto de algunas variables claves (como la tasa de interés) sobre la tasa de ahorro. Sin embargo, es muy infrecuente que se mencione, aunque sea como hipótesis muy tentativa, la posible influencia de factores puramente culturales en el ahorro, en concordancia con la "aversión" de la profesión a aceptar la relevancia de este tipo de variables. $\mathrm{Al}$ reconocer que éstos desempeñan un papel, surge como evidente la necesidad de combinar los "incentivos materiales" para 
aumentar el ahorro con una política comunicacional que apunte a modificar las pautas de consumo de las familias latinoamericanas. De algún modo, este argumento reivindica lo que Prebisch consideraba que era uno de los factores que retardaban el crecimiento de América Latina: la imitación de pautas de consumo originarias de sociedades con un ingreso per cápita muy superior al de la región.

Por otro lado, es frecuente que en los trabajos orientados a la formulación de políticas se hagan afirmaciones que, si bien están respaldadas por la literatura teórica y despiertan consenso entre los economistas, deberían expresarse con mayor cautela. Por ejemplo, se suele señalar la importancia de un entorno macroeconómico estable como requisito necesario de cualquier estrategia destinada a elevar el ahorro, y hacer hincapié en los beneficios de contar con una gama diversificada de instrumentos financieros. Sin embargo, en los Estados Unidos, donde no sólo se cumple con dicho requisito sino que además los agentes disponen de un número mayor de vehículos para el ahorro que en cualquier otra economía, la tasa de ahorro de las familias ha sido negativa en los últimos años. Paradójicamente (desde un punto de vista convencional), hay evidencia de circunstancias en que el ahorro de las familias reacciona positivamente ante aumentos de la incertidumbre.

No parece ser tarea sencilla estimular el ahorro de las familias en América Latina, basándose exclusivamente en incentivos de mercado. Este ahorro es muy bajo (y en ocasiones negativo). De hecho, en nuestra región el ahorro proviene mayoritariamente del sector empresas y en una proporción superior a la que se observa en economías desarrolladas. Pese a esta dificultad, no cabe duda de que el incremento del ahorro privado en América Latina exige ineludiblemente que se fomente el ahorro de las familias.

\section{a) El sistema previsional}

En primer lugar, es práctica de rutina afirmar que un sistema previsional desfinanciado tiene un impacto negativo sobre el ahorro. Esto es cierto en primera instancia. Pero no debe olvidarse que, en definitiva, lo que importa no es donde aparecen anotados los desequilibrios en las cuentas públicas sino la magnitud global del déficit fiscal. Se puede diseñar un sistema previsional de "reparto" plenamente financiado a través de mayores aportes y contribuciones (con lo cual, según el argumento comentado, no habría impacto negativo sobre el ahorro). Si aceptamos que toda economía, según sus características, soporta un cierto ni- vel máximo de presión tributaria total, la contrapartida del equilibrio del sistema previsional conseguido de esta manera sería un mayor desequilibrio en otros rubros de las cuentas públicas, dado que el nivel de los tributos no previsionales debería ser menor que si los aportes previsionales fueran bajos.

En segundo lugar, pareciera que en el contexto latinoamericano, incluso sin exagerar el grado de racionalidad de los agentes, los futuros beneficiarios del sistema previsional suelen aplicar una tasa de descuento alta a sus futuros ingresos, ya que la probabilidad de que no pueda cumplirse cabalmente con las promesas incorporadas en las leyes previsionales vigentes es alta. En el mismo sentido de lo aquí afirmado, las autoridades deberían procurar que la población tome conciencia de la necesidad imperiosa de complementar con ahorros adicionales los ingresos que provendrán del régimen previsional.

Una posición que ha ganado creciente apoyo es la que considera conveniente implementar sistemas de capitalización individual, como los ya adoptados en varios países latinoamericanos, basándose en el estímulo que tales esquemas ejercen sobre el desarrollo del mercado de capitales. Cabe señalar que, en la práctica, esta conexión es más débil que lo que sugiere la literatura y que la evidencia empírica no muestra un impacto dinámico como el que a priori esperaban los propulsores de las reformas de los sistemas previsionales en el decenio de 1990.

Gran parte de los activos de los sistemas de capitalización son invertidos en títulos públicos emitidos por los gobiernos para financiar, principalmente, el desequilibrio provocado por la transición de un sistema a otro. En algunos casos, estos bonos pagan tasas de interés significativas que plantean dudas sobre la solvencia intertemporal del sector público.

Una posible solución intermedia entre los dos modelos polares es un régimen de capitalización, pero administrado por el Estado. El costo de administración de los sistemas de capitalización privados resulta muy alto, además de tener indeseables consecuencias distributivas debido a la estructura de las comisiones cobradas por las administradoras de fondos de pensión (AFP). Las primeras experiencias en América Latina muestran que estos regímenes son ineficientes. Dado que la competencia entre AFP se realiza sobre la base de la publicidad, y no de diferencias de desempeño, se están utilizando recursos sociales escasos para una actividad estéril que carece de contenido informativo real para guiar de manera racional las decisiones de los contribuyentes. 
A esto cabe agregar los costos de la regulación y supervisión del sistema. En realidad, puede incluso argumentarse que éste no sería eficiente ni siquiera si los agentes se guiaran sólo por los rendimientos que hayan obtenido las administradoras. Esto es así porque los marcos regulatorios hacen que el margen para diferenciar carteras es bajo y porque un buen desempeño en el pasado no siempre significa más altos rendimientos en el futuro. Llevando el argumento al extremo, en mercados aproximadamente eficientes la probabilidad de obtener rendimientos excepcionales es necesariamente baja y para el sistema en su conjunto es inexistente.

Por los motivos mencionados se puede argumentar que un sistema de capitalización estatal es tal vez el óptimo. Pero las dificultades para implementarlo en el contexto latinoamericano no son menores. Las principales objeciones tienen que ver con su potencial vulnerabilidad a eventuales presiones del gobierno y de grupos de interés. Precisamente, esa vulnerabilidad es una de las razones que explican la inviabilidad de los antiguos sistemas de reparto. Pero debería plantearse la posibilidad de otorgar a tal régimen estatal un grado de independencia similar al que tienen algunos bancos centrales, con autoridades cuyo mandato excede los períodos de gobierno de las autoridades políticas. Cabe señalar que una alta participación de la deuda pública en la cartera del sistema de capitalización administrado por el gobierno no invalida su "optimalidad", ya que esa alta proporción se observa también en el sistema privado.

Si de todos modos hubiera consenso para proseguir en el camino de reemplazar los sistemas de reparto por sistemas de capitalización privada, es imperativo mejorar el funcionamiento de estos últimos. Hay dos aspectos en los que es necesario avanzar urgentemente. Primero, hay que mejorar los incentivos para disminuir aquellos gastos administrativos que no redundan en mejores servicios a los clientes. En segundo lugar, ha habido situaciones y comportamientos en que se plantearon agudos conflictos de intereses, que implicaron perjuicios para los ahorristas y que fueron motivados por la falta de una adecuada separación entre las AFP y los bancos. En algunos casos las AFP fueron "presionadas" a adquirir paquetes accionarios a precios más altos que los de mercado, aprovechando circunstancias como una fusión o la creación de una estructura de holding. Y también ha habido casos de bancos que "descargaron" activos indeseables en las AFP, incluso utilizando la triangulación con otras entidades.
Estos problemas, derivados de la inexistencia o debilidad de muros divisorios y que por su alcance exceden el ámbito del tema puramente previsional, se han dado incluso en contextos en que el cumplimiento estricto de la legislación vigente (mayoritariamente "importada" de países con buenas prácticas) no permitía las operaciones a las que nos referimos.

Lo dicho destaca la importancia de que se exija el cumplimiento de las normas ya sancionadas, lo que parece ser débil en la región. En particular, los organismos reguladores establecidos por los gobiernos muestran una tendencia a ser "capturados" por las instituciones reguladas de mayor peso. Por lo tanto, es imprescindible reforzar la estabilidad de los funcionarios de los entes reguladores y garantizar su invulnerabilidad a las decisiones del poder político; por otro lado, es preciso impedir que los funcionarios que dejan los entes reguladores pasen inmediatamente a trabajar para las empresas que regulaban, sin que medie un período de transición.

Un elemento que podría contribuir a elevar la tasa de ahorro es la incorporación de cotizaciones voluntarias adicionales, pero con características de liquidez que las tornen más atractivas para los futuros pensionados. La ausencia de este factor parece explicar en buena medida el bajo nivel de los aportes adicionales en los sistemas que los permiten.

\section{b) Ahorro de las empresas}

Hemos señalado más atrás que el ahorro en América Latina proviene básicamente del sector empresas. A nivel agregado, se observa que la retención de utilidades como fuente de fondos de las empresas tiene un peso mayor en los países de la región que en los países desarrollados. Además de recurrir a incentivos tributarios y normas contables que desalienten la distribución de dividendos, se puede fomentar el ahorro de las empresas con políticas que estimulen la inversión, ya que una alta rentabilidad de los proyectos opera como un incentivo "natural" a la reinversión de utilidades. Esto se debe a que el uso de fondos propios es la alternativa de más bajo costo, como lo muestra tanto la literatura teórica en el campo de las finanzas como la evidencia empírica de países industrializados y de menor desarrollo relativo. En efecto, las empresas recurren al endeudamiento bancario, a la colocación de bonos o a la emisión de acciones nuevas cuando los fondos internos son insuficientes. Esta brecha entre el costo del financiamiento interno y externo, que constituye la prima de financiamiento externo, es más alta en los países latinoamericanos, 
probablemente porque en ellos los problemas de información asimétrica son más graves.

Pareciera que las empresas en América Latina están endeudadas por debajo de los niveles óptimos (se encuentran "subapalancadas"), lo cual es coherente con la existencia de fallas de mercado en el sistema financiero. Esto puede resultar a primera vista paradójico, ya que en la mayoría de los sistemas tributarios de la región existen fuertes incentivos para endeudarse. Esto pone de relieve la necesidad de aumentar la profundidad financiera de la economía y desarrollar el mercado de capitales para estimular el ahorro y canalizarlo hacia las empresas con proyectos más rentables. Pero una política en tal sentido debe tratar de compensar el sesgo en contra de las PYME provocado por todo sistema que induzca al endeudamiento empresarial. Es clave procurar que las PYME tengan mejor acceso al crédito.

\section{c) Ahorro del sector público}

Una política fiscal prudente no es sinónimo de superávit fiscal o de déficit fiscal nulo, ya que un país en crecimiento puede financiar déficit moderados sin que hacerlo sea necesariamente desestabilizante. Por otra parte, la recomendación de alcanzar un superávit o equilibrio fiscal está formulada en términos muy generales y trata a los países de la región como si el conjunto tuviera una homogeneidad de la cual carece. La imposición indiscriminada de metas de superávit fiscal es excesivamente voluntarista y puede resultar negativa, incluso en términos de la solvencia fiscal intertemporal. Las diferencias entre las instituciones fiscales y también de organización política entre los países, implican dispares grados de libertad para el ejercicio de la política fiscal. Estas restricciones, que son especialmente relevantes en países grandes y federales, habrán de ser tomadas en cuenta eventualmente para formular propuestas de reforma (la regionalización, por ejemplo) que apunten a la raíz del problema. Hay que tener presente, por otro lado, que ante la falta de cierta libertad para manejar las políticas monetaria y cambiaria es difícil justificar la autoimposición de un límite al superávit presupuestario, que en la práctica dejaría a los países en la incapacidad de moderar siquiera mínimamente las perturbaciones que afecten a sus economías.

Asimismo, es indudable la necesidad de liberar recursos (ahorro) para la inversión privada. Sin embargo, en América Latina no faltan proyectos con alta rentabilidad social, superior incluso a la de los proyec- tos que puede encarar el sector privado con la liberación de más recursos por parte del sector público.

Por otra parte, a veces los gobiernos hacen esfuerzos heroicos de ajuste fiscal para alcanzar metas fiscales incorporadas en acuerdos con el FMI; esto los fuerza a realizar operaciones que generan costos asociados, los que pueden ser considerables en el largo plazo. Un ejemplo es la venta de empresas públicas o paquetes accionarios, o el otorgamiento de concesiones, sin esperar las circunstancias más propicias para llevarlas a cabo, por el afán de cumplir con la letra de las metas fiscales asumidas. Esto no permite aprovechar el valor potencial de la renta obtenible por el sector privado y en realidad atenta contra la solvencia fiscal de largo plazo, en la medida en que el valor presente de los ingresos públicos resulta menor.

En otro plano, es frecuente que el sector público cumpla un papel adicional: el de obtener las divisas necesarias para financiar el déficit de cuenta corriente. En efecto, en ciertos países de la región el sector privado es estructuralmente deficitario en sus operaciones externas y su contribución a la acumulación de reservas es negativa, mientras que el sector público obtiene recursos en divisas por encima de sus necesidades financieras externas y sí contribuye a la acumulación de reservas y al financiamiento del déficit externo del sector privado. En este sentido, una reducción del déficit fiscal podría implicar una depreciación de la moneda y/o dificultades para financiar el déficit de cuenta corriente del sector privado.

\section{d) Los usos del ahorro}

El esfuerzo realizado en la última década para ampliar la variedad de instrumentos de ahorro emitidos por residentes y los incentivos (tributarios y de regulación) adoptados para promover su adquisición en los mercados latinoamericanos no ha producido los efectos esperados. En la región hay numerosos ejemplos de aumento de los ahorros financieros mientras se mantuvo o cayó la tasa de ahorro. En muchas experiencias de desarrollo exitosas el consumo de ciertos tipos de bienes sólo recibió impulso una vez alcanzado un nivel de desarrollo considerable. Por ejemplo, en América Latina las importaciones de bienes de consumo durables tienen un importante efecto negativo en el balance de pagos. En este caso, la aplicación de determinadas restricciones (por ejemplo, tarifarias) contribuye al aumento del ahorro.

Aparte de los aspectos vinculados con el financiamiento de la inversión, un tema de primordial importancia es la necesidad de aumentar la eficiencia o productividad de la inversión y mejorar el muy débil 
mantenimiento del capital social que se observa en nuestros países (lo que implica una alta tasa de depreciación). Ciertamente en América Latina hay un amplio espacio para ganar con un mejor mantenimiento, aun para una tasa de inversión neta dada.

\section{La intermediación financiera}

\section{a) La concentración bancaria}

Cabe preguntarse si la tendencia a una mayor concentración bancaria no debiera ser motivo de preocupación. La respuesta dista de ser categórica. No todos los sistemas financieros de la región exhiben un nivel de concentración importante. Además, el deseo de reducir ese nivel puede chocar con otros objetivos, como el de mantener una participación considerable de los bancos nacionales. En efecto, en un contexto como el actual, en que la globalización en el terreno financiero avanza a pasos agigantados, las entidades locales sólo pueden mantener una participación significativa adquiriendo un tamaño importante que les permita competir con los bancos extranjeros y/o que opere como barrera a la entrada de potenciales competidores. En definitiva, el problema del riesgo moral derivado de la presencia de entidades que son "demasiado grandes para fracasar" (y tienen, por lo tanto, un seguro implícito) debe ser atacado mediante el fortalecimiento del marco regulatorio y de la supervisión bancaria. El tamaño de los bancos no constituye un problema per se (como lo prueba el caso canadiense, entre otros), a menos que se combine con un marco institucional muy débil.

Tampoco se justifica una exagerada preocupación por los riesgos de una mayor concentración bancaria si ésta se debe a una mayor participación de entidades extranjeras, especialmente si se trata de bancos internacionales de primera línea. El riesgo moral es menor en este caso, ya que es menos probable, y menos tolerable políticamente, que los gobiernos acudan en socorro de bancos extranjeros antes que de bancos públicos o privados nacionales. En este sentido, puede argumentarse que los riesgos de la concentración no son independientes de la nacionalidad de los bancos.

\section{b) Mayor participación de entidades foráneas}

Si bien en principio - a un nivel alto de generalidad - la mayor presencia de instituciones financieras extranjeras aumenta la correlación internacional de riesgos sistémicos, esto no parece tener mucha importancia en el contexto latinoamericano. Evitar la "importación" de crisis bancarias desde los países desarro- llados no es, ni debería ser, una preocupación prioritaria en nuestros países, donde hay fuentes locales de perturbación mucho más importantes. Aunque en teoría la correlación mencionada aumenta, en la región esto se asocia a una moderación del riesgo ya que, en general, los grandes bancos extranjeros son percibidos como más solventes que los locales.

Esto es así, primero, porque los bancos entrantes están en general más cerca de las "mejores prácticas" financieras que los bancos latinoamericanos. Segundo, porque (hasta cierto punto) las casas matrices de los bancos entrantes pasan a actuar como prestamistas de última instancia de sus filiales locales. En la práctica se ha observado incluso que un banco grande de un mercado emergente de América Latina (Banamex) capitalizó un banco controlado que opera en Argentina (Bansud) y que atravesaba una situación muy comprometida, con tal de no asumir los costos de reputación que implicaba su quiebra. Por lo tanto, es de esperar que bancos cuyas casas matrices se encuentran en mercados más desarrollados actúen con similar o mayor esmero en la solución de los problemas que puedan enfrentar sus filiales en esta región.

Finalmente, desde otra perspectiva, la presencia de estos bancos permite a los depositantes diversificar su cartera entre instituciones con alcance internacional, lo cual reduce el riesgo-país que asumen. Esta es una alternativa preferible a que la reducción del riesgo de los inversores locales se efectúe a través de la fuga de capitales.

En general, la experiencia en América Latina parece indicar que en la región es preferible contar con un sistema de bancos universales bastante concentrado. Ha habido casos en que la competencia dentro de marcos regulatorios débiles llevó a las instituciones bancarias a adoptar estrategias demasiado arriesgadas que potenciaron la inestabilidad del sistema financiero. En cambio, una tasa de ganancia más alta que la de competencia reduce la probabilidad de corridas bancarias y los incentivos a enfrentar riesgos. Asimismo, un sistema de banca universal con predominio de entidades grandes puede contribuir a mejorar la calidad de la gestión y la gobernabilidad de esas instituciones.

No obstante, lo afirmado no significa que el proceso de concentración bancaria y la mayor participación de entidades extranjeras no tenga derivaciones negativas que sea necesario vigilar activamente. En primer lugar, una excesiva concentración u oligopolización puede permitir que las entidades dominantes generen elevadas cuasi rentas; en ese caso, la intervención 
estatal se justificaría por la necesidad de corregir esa falla de mercado y necesitaría un marco regulatorio que estipulara porcentajes máximos de participación e impidiera fusiones que generaran obstáculos a la competencia. Pero también puede justificar el papel de los bancos públicos de "primer piso" o minoristas, en la medida en que puedan operar como bancos "testigo", obligando a los bancos privados a fijar precios más cercanos a los que se darían en un contexto más competitivo.

En segundo lugar, por lo general los bancos más grandes (particularmente los extranjeros) siguen prácticas más conservadoras en el otorgamiento de créditos. Es frecuente, además, que bancos regionales o que atienden a sectores de considerable riesgo sean reemplazados en ciertas localidades por estos bancos internacionales, lo cual reduce de manera drástica la oferta de crédito, precisamente para empresas o individuos con grandes dificultades para mantener un acceso fluido al financiamiento (actividad agropecuaria, pequeñas y medianas empresas, emprendimientos en regiones desfavorecidas, familias de ingresos bajos o medios/bajos, etc.). Por lo demás, cabe señalar que el nivel de delegación de responsabilidades de crédito disminuye con la mayor participación de bancos extranjeros. En la práctica, todo crédito de montos significativos, particularmente los vinculados con el financiamiento de proyectos de inversión, son aprobados en el país donde el banco tiene su casa matriz. Este es claramente un resultado indeseable de la mayor participación de bancos extranjeros y sugiere que es necesario asegurar la existencia de una banca local fuerte, ya sea pública o privada. Por otro lado, si bien es difícil cuantificar la magnitud de este efecto, desde un punto de vista macroeconómico la concentración de la responsabilidad del crédito en la casa matriz aumenta la correlación del ciclo entre los países latinoamericanos y entre éstos y los países de origen de los bancos extranjeros. La sensibilidad de la inversión a la prima de riesgo también aumentaría.

\section{c) La banca pública en el contexto actual}

Por todos los motivos indicados más arriba, el papel central que le cabe a la banca pública no debe ser subestimado. Pero indudablemente, la forma en que dicha banca debe cumplir su papel tiene que ser adaptada al nuevo contexto internacional y local. En varios países de la región, muchos de los principales bancos comerciales continúan en manos del Estado, el que mantiene una posición clave en los sistemas financieros domésticos. La banca pública tuvo un papel deci- sivo en América Latina durante el período de industrialización a través de la sustitución de importaciones (III). El crédito dirigido aumentó rápidamente, abarcando a muchos sectores, y se convirtió en una herramienta preeminente para apoyar el desarrollo de la industria, la agricultura y los programas sociales. Los bancos públicos intentaron suplir las deficiencias de los débiles mercados de capitales internos como fuente de financiamiento de largo plazo, pero graves problemas de gestión los llevaron a acumular enormes quebrantos, que aún persisten a pesar de que las políticas de crédito dirigido se han tornado más focalizadas y más modestas.

Los bancos públicos, en su inmensa mayoría, no han dado a la evaluación de los riesgos crediticios la importancia que merece, y exhiben niveles de recuperación de créditos alarmantemente bajos y tasas de morosidad extraordinariamente elevadas. Estas instituciones fueron muy vulnerables a las presiones de los grupos de interés y, en muchos casos, víctimas del afán de lucro. En suma, se convirtieron en un mecanismo extremadamente ineficiente de canalización de subsidios. Incluso en países desarrollados ha habido experiencias costosas con bancos públicos de primer piso. Un caso reciente es el del Crédit Lyonnais, en Francia, que ha exhibido quebrantos importantes, a pesar del alto grado de profesionalismo de su personal.

Teniendo en cuenta estas consideraciones, ¿cuál debería ser la función de la banca estatal en América Latina? Tanto la teoría económica como las experiencias del este de Asia sugieren que los programas de crédito focalizados y bien administrados pueden funcionar bien en muchos casos. Pero cabe preguntarse cuáles son los canales más idóneos para llevar adelante tal política. Dados los importantes efectos adversos que provoca el mal funcionamiento de la banca estatal de primer piso, parecería imperativo en muchos casos transformar la banca estatal en banca de segundo piso. La banca estatal de primer piso estaría justificada ante la ausencia o insuficiencia de una adecuada atención de la banca privada en determinadas regiones (presumiblemente por la baja rentabilidad de éstas), o bien frente a una segmentación que castigue a las PYME, o si el sistema financiero privado es fuertemente oligopólico y la banca estatal pudiera operar como un efectivo banco testigo. Existe, además, un papel adicional que la banca pública puede cumplir bajo algunos diseños institucionales del sistema financiero (por ejemplo, en un régimen de convertibilidad, cuando el banco central está limitado en su función de prestamista de última instancia). 
En cualquier caso, la subsistencia de la banca estatal, mayorista y minorista, sólo puede justificarse si se encaran importantes reformas en el gobierno de estas instituciones. Es necesario que la banca estatal de primer piso se ciña a las normas de regulación, supervisión y calificación de riesgo que rigen en los bancos privados. Pero, además, es necesario evitar la superposición inútil de bancos estatales que "compiten" en los mismos mercados: esto es ineficiente y significa un desperdicio de recursos sociales. La necesidad de contar con un banco testigo o que actúe de manera contracíclica cuando la banca privada exhiba gran aversión al riesgo no implica que deba haber numerosas entidades bancarias públicas. Su multiplicidad crearía el riesgo de que surgiera entre ellas una competencia estéril por réditos de tipo político.

Hay algunos principios básicos que la banca pública debería observar y que, en muchos casos, exigiría un considerable rediseño institucional. Primero, todo componente de subsidio implícito en las prácticas crediticias de estos bancos debe mostrarse en forma transparente y ser presupuestado. La valuación correcta de los subsidios es un requisito necesario para que los contribuyentes tomen conciencia del costo de mantenimiento de estas instituciones $y$, por otra parte, permitiría que las cámaras legislativas, encargadas de aprobar el presupuesto gubernamental, ponderaran adecuadamente su peso dentro del orden de prioridades de la política gubernamental.

Segundo, los bancos públicos, al igual que el banco central, deben ser completamente independientes de los poderes políticos. A fin de garantizar esa independencia, los miembros de su directorio deben tener un período de mandato más largo que el de las autoridades del Poder Ejecutivo. Asimismo, los directorios de estas instituciones deben incorporar directores por la minoría política que sirvan como mecanismo de control y auditoría. Por otra parte, una vez alcanzado cierto nivel de transparencia en las cuentas de estos bancos, se podría establecer sistemas de incentivos para el directorio y la alta gerencia vinculados con el desempeño.

Tercero, la banca pública o de desarrollo debe dar prioridad al financiamiento de proyectos vinculados con la producción de bienes transables que permitan incrementar las exportaciones o sustituir importaciones. Por lo tanto, habría que elaborar una lista jerarquizada que ordene los proyectos presentados según su capacidad de generar divisas. En principio, los sectores que no puedan generar ni ahorrar divisas no deberían recibir mucha asistencia crediticia de parte de esta banca, y deberían buscarla en el sector privado.
Cuarto, un capítulo especial merece el sector de las PYME. Para estas empresas se podría justificar un componente de subsidio, aun tratándose de actividades no transables, si su potencial de generación de empleo estuviese muy por encima del promedio. En este caso, tal vez convenga subsidiar con fondos presupuestarios la tasa de interés activa para los préstamos a las PYME, distribuyendo el subsidio entre los bancos de acuerdo con la menor tasa activa propuesta. Este tipo de instrumento es muy eficiente, dado que tiene una alta eficacia con una erogación relativamente pequeña. El arriendo con opción de compra (leasing) es otro instrumento que puede ser muy útil para contrarrestar los efectos de las altas tasas de interés y la exigencia de garantías reales excesivas para otorgar a las PYME préstamos destinados a la adquisición de maquinaria. En muchos países de América Latina el marco jurídico no es del todo adecuado para este tipo de operación y debería ser reformado. En particular, sería conveniente exigir a los bancos que establezcan filiales separadas para hacer contratos de leasing. También sería deseable que se permitiera la amortización del activo (para el locatario) en un plazo igual o menor que el período de vigencia del contrato de leasing. Además, para que este tipo de contrato resulte atractivo es casi imprescindible que los pagos de las cuotas sean deducibles de impuestos para el locador. Por el lado de la demanda, la experiencia indica que un aspecto de importancia no menor es el de preparar la carpeta de crédito para el banco. Al respecto puede pensarse en impulsar en toda la región un sistema de consultoría al modo chileno (es decir, el de subsidiar parte del costo de la compra de servicios a profesionales habilitados con ese fin por el Estado), o bien en recurrir a un esquema de presentación simplificada como el de la Small Business Administration de los Estados Unidos. En este último caso, el formulario simplificado se podría concordar con los bancos. Ambos esquemas pueden complementarse si la consultoría no se restringe a la tramitación del crédito sino que comprende también la gestión del proyecto para el cual se pide el préstamo.

Hay un consenso generalizado acerca de la conveniencia de impulsar o reforzar las Sociedades de Garantías Recíprocas (SGR). En los países de mayor tamaño de la región, donde cabe esperar un número significativo de operaciones (lo cual disminuiría el costo medio de administración) también puede considerarse la alternativa de un Fondo Público de Garantía. Si se opta por un sistema mixto, este fondo deberá actuar como entidad prestadora de contragarantías, haciéndose 
cargo de parte del riesgo asumido por las SGR. Hay que tener presente que la existencia de un Fondo Público de Garantía no debe, en ningún caso, eliminar la necesidad de que las SGR demuestren su eficacia a través de una gestión de estricto rigor profesional a la hora de evaluar proyectos. En este sentido, podría pensarse en un sistema en el cual el costo del reaseguramiento sea inversamente proporcional a la eficacia de la SGR, medida sobre la base del índice de falencia de las operaciones avaladas.

Otros instrumentos que deberían estimularse son los Fondos de Riesgo y la figura del Fideicomiso de Crédito. Con ellos se puede dar mayor eficiencia a la utilización de los recursos públicos disponibles y aprovechar mejor la capacidad prestable, gracias a la participación de grandes inversores institucionales del sector privado. Para optimizar el uso de los fideicomisos es conveniente focalizarlos en microempresas y en pequeños emprendimientos, ya que los proyectos de esta índole son los que tienen más dificultades para acceder al sistema bancario y, por lo tanto, carecen de alternativas de financiamiento. En lo que se refiere a las microfinanzas, no debería subestimarse el papel de las cooperativas de ahorro y crédito. Si bien la experiencia regional es matizada, pues exhibe algunos notorios éxitos y fracasos, estas instituciones son especialmente aptas para brindar financiamiento a las PYME y a los sectores de menores ingresos, al mismo tiempo que consiguen niveles satisfactorios de recuperación de los créditos. Pero es necesario adaptar las normas regulatorias y el marco legal para asegurar que puedan sostener la competencia con los bancos comerciales y para solucionar los problemas de gobernabilidad que frecuentemente las aquejan.

Quinto, en la medida en que sea posible, la banca pública debe compartir el financiamiento con la banca privada. Es decir, se debe tratar de evitar una participación excesiva de la primera en la financiación de los proyectos. Lo ideal sería reducir la financiación pública al mínimo pero procurando compartir parte del riesgo (no así el financiamiento) del proyecto a través de un sistema de garantías. 